\title{
Cementos compuestos elaborados con puzolana natural y escoria granulada de alto horno
}

\section{Composite cements containing natural pozzolan and granulated blast furnace slag}

\author{
V. L. Bonavetti ${ }^{(*)}$, G. Menéndez(*), H. A. Donza(*), V. F. Rahhal(*), E. F. Irassar(*)
}

Recepción/Received: 6-VII-05

Aceptación/Accepted: 20-II-06

\section{RESUMEN}

Debido a las exigencias del mercado y de la producción de cemento Portland, es cada vez más frecuente la elaboración de cementos con dos o más adiciones a partir de la molienda separada de sus constituyentes, dando origen a la formulación de los cementos a medida.

Cuando el contenido de adiciones es alto, la utilización de puzolana y escoria en este tipo de cementos presenta la peculiaridad de disminuir la resistencia inicial del cemento resultante. Sin embargo, si algunas o ambas adiciones se activan, este problema puede minimizarse.

En este trabajo se analiza la influencia de la incorporación al cemento Portland de puzolana natural (hasta $20 \%$ ) activada físicamente y/o escoria granulada de alto horno (hasta $35 \%$ ) sobre la resistencia a flexión y a compresión de morteros. Los resultados indican que los cementos ternarios presentan un mejor comportamiento resistente que los cementos binarios. Las máximas resistencias a compresión en las primeras edades ( 2 y 7 días) se alcanzan con hasta $13 \%$ de puzolana natural y $5 \%$ de escoria, mientras que a edades más avanzadas se trasladan a mayores contenidos de puzolana y escoria.

Palabras clave: cemento Portland, puzolana, escoria granulada de alto horno, resistencia a compresión, resistencia a flexión.

\section{SUMMARY}

For reasons of market demand and Portland cement production, the manufacture of cements with two or more separately ground additions to produce customized cements is becoming common practice.

When pozzolan or slag content in this type of cements is high, however, the initial strength of the resulting product may be adversely impacted. This problem can be minimized by activating one or both of the replacement materials.

The present study analyzes the effect of Portland cement additions such as physically activated natural pozzolan (up to 20\%) and/or granulated blast furnace slag (up to $35 \%$ ) on mortar flexural and compressive strength. The results show that higher strength is attained in ternary than binary cements. Initially ( 2 and 7 days), the highest compressive strengths are reached by mortars with up to $13 \%$ natural pozzolan and 5\% slag, whereas at later ages mortars with larger proportions of additions are found to perform best.

Keywords: Portland cement, pozzolan, granulated blast furnace slag, compressive strength, flexural strength.

(*) Departamento de Ingenería Civil. Universidad Nacional del Centro, Argentina. 


\section{INTRODUCCIÓN}

La producción de cementos con dos o más adiciones a partir de la molienda separada de sus constituyentes (cementos a medida) permite la elaboración de cementos con una amplia variedad y porcentaje de adiciones, sin aumentar la complejidad del sistema de producción, en función de los cuales se pueden obtener las propiedades resistentes y/o durables preestablecidas. Actualmente son muy conocidos los beneficios tecnológicos, económicos, y el menor impacto medioambiental que produce el empleo en forma aislada de puzolana natural y de escoria granulada de alto horno como reemplazo parcial de cemento Portland. Sin embargo, la influencia conjunta de estas adiciones sobre el mecanismo de hidratación y, en consecuencia, sobre las propiedades mecánicas de los cementos compuestos resultantes aún no está bien establecida.

Es conocido que el desarrollo de la resistencia de un cemento con adiciones hidráulicamente activas depende del efecto físico y de la reacción de la adición. Las partículas de escoria y puzolana son generalmente menos reactivas que las del cemento Portland. Pero cuando estas partículas, finamente molidas, se dispersan dentro de la matriz cementante, producen un gran número de sitios de nucleación para la precipitación de los productos de hidratación del cemento Portland, acelerando su resistencia inicial. Este efecto físico, denominado efecto filler, tiende a disminuir la pérdida de resistencia a edad temprana del cemento compuesto, dado por la menor cantidad de material inicialmente reactivo (1). Las partículas de escoria o puzolana reaccionan con los hidróxidos alcalinos generando un gradual decrecimiento del $\mathrm{CH}$ y un incremento del $\mathrm{CSH}$ y de aluminosilicatos de calcio similares a los obtenidos durante la reacción del cemento Portland (2-5). Como consecuencia de esta reacción química, se produce el refinamiento de los granos y la segmentación de los poros, aumentando la densidad de la matriz, la resistencia y la inmunidad de la pasta frente al ingreso de los agentes agresivos (6).

Por otra parte, debido a la baja reactividad inicial de estas adiciones, los cementos con escoria y puzolana presentan una disminución de la resistencia inicial, que suele limitar su empleo. Pero, este inconveniente puede mitigarse a partir de la activación de una o ambas adiciones. Así, se pueden obtener adecuadas resistencias iniciales con la activación física, por el aumento en la finura de la adición (6), la activación térmica, por el incremento en la temperatura de curado (7), y la activación química, por la incorporación de hidróxidos alcalinos a la mezcla (8).

Por último, en investigaciones previas se ha analizado la influencia del empleo conjunto de escoria granulada de

\section{INTRODUCTION}

The manufacture of cements with two or more separately ground components (customized cements) is a way of broadening the variety and percentage of additions to achieve certain pre-established strength and/or durability properties without increasing the complexity of the production system. The technological, economic and environmental benefits of the separate use of natural pozzolan and granulated blast furnace slag as partial replacements for Portland cement are well known at this time. Nonetheless, the joint effect of these additions on the hydration mechanism and consequently the mechanical properties of the resulting composite cements have not yet been clearly established.

Strength development in a cement with hydraulically active additions is known to depend on the physical effect and reactivity of the latter. Slag and pozzolan particles are generally less reactive than Portland cement particles. But when finely ground and mixed with the cementitious matrix, they generate a large number of nucleation sites for the precipitation of Portland cement products, accelerating initial strength development. Known as the filler effect, this development tends to reduce the loss of early age strength in composite cement occasioned by the smaller proportion of initially reactive material (1). As in Portland cement reactions, in the reaction between pozzolan or slag particles and alkaline hydroxides, $\mathrm{CH}$ content gradually decreases while the $\mathrm{CSH}$ and calcium aluminosilicate concentration increases (2-5). This chemical reaction prompts particle refinement and pore segmentation, enhancing matrix density, strength and paste immunity to aggressive agents (6).

By contrast, due to the low initial reactivity of slag and pozzolan, cements with these additions have lower initial strength, which tends to limit their use. But this drawback can be mitigated by activating one or both additions. Good initial strength can be obtained, then, with physical activation, by grinding additions more finely (6), thermal activation, by raising curing temperature (7) or chemical activation, by adding alkaline hydroxides to the blend (8).

Lastly, prior research has been conducted on the effect of the joint use of granulated blast furnace slag and a 
alto horno y filler calcáreo $(9,10)$ y de puzolana natural y filler calcáreo (11) sobre la resistencia y el agua no evaporable de morteros; mientras que en el presente trabajo se estudia la evolución de la resistencia y la puzolanicidad de morteros elaborados con cemento Portland con reemplazos de puzolana natural activada físicamente (hasta 20\%) y/o escoria granulada de alto horno (hasta 35\%).

\section{PARTE EXPERIMENTAL}

Cemento: para la realización de los ensayos se utilizó un cemento Portland sin adiciones [CEM I, EN 1971:2000 (12)], con una composición mineralógica de $58 \%$ de $\mathrm{C}_{3} \mathrm{~S}, 18 \%$ de $\mathrm{C}_{2} \mathrm{~S}, 2 \%$ de $\mathrm{C}_{3} \mathrm{~A}$ y $13 \%$ de $\mathrm{C}_{4} \mathrm{AF}$ y un contenido de yeso de 5,85\%, categoría resistente 42,5 y finura Blaine de $321 \mathrm{~m}^{2} / \mathrm{kg}$. La distribución del tamaño de las partículas presentó un parámetro posición $\left(\chi^{\prime}\right)$ de 28,81 um y un parámetro de homogeneidad (n) de 0,93 (13). En la Tabla 1 se muestra la composición química del cemento.

Adiciones: como adiciones se emplearon escoria granulada de alto horno y puzolana natural. La escoria granulada de alto horno (E) con una finura Blaine de $458 \mathrm{~m}^{2} / \mathrm{kg}$ presentó un módulo químico $\left[\left(\mathrm{CaO}+\mathrm{MgO}+\mathrm{Al}_{2} \mathrm{O}_{3}\right) / \mathrm{SiO}_{2}\right]$ de 1,8 y su análisis por DRX mostró sólo una pequeña proporción de materiales cristalinos (melilita). La puzolana natural $(P)$ con finura Blaine de $760 \mathrm{~m}^{2} / \mathrm{kg}$ estaba constituida por $93 \%$ de vidrio volcánico ácido y sus principales materiales cristalinos determinados por DRX fueron: cuarzo y feldespatos (anortita, albita, chabazita y analcita). En la Tabla 1 se informa de la composición química de las adiciones.

Con el objeto de producir un aumento del efecto físico inicial y la activación química de la puzolana, que permitiera calcareous filler $(9,10)$, and natural pozzolan and a calcareous filler (11) on mortar strength and non-evaporable water; the present study analyzes strength development and pozzolanicity in mortars made with Portland cement partially replaced by physically activated natural pozzolan (up to 20\%) and/or granulated blast furnace slag (up to $35 \%$ ).

\section{EXPERIMENTAL}

Cement: the tests were run with an unadditioned, class 42.5 Portland cement (European standard EN 1971:2000 CEM I) with a Blaine fineness of $321 \mathrm{~m} / \mathrm{kg}$ and mineral composition: $58 \% C_{3} S, 18 \% C_{2} S, 2 \% C_{3} A 13 \%$ $C_{4} A F$ and $5.85 \%$ gypsum. The particle size distribution position parameter $\left(\chi^{\prime}\right)$ was $28.81 \mu \mathrm{m}$ and the homogeneity parameter (n) 0.93 (13). The chemical composition of the cement is given in Table 1.

Additions: natural pozzolan and granulated blast furnace slag were the additions used. The chemical modulus $\left[\left(\mathrm{CaO}+\mathrm{MgO}+\mathrm{Al}_{2} \mathrm{O}_{3}\right) / \mathrm{SiO}_{2}\right]$ of the granulated blast furnace slag (E) was 1.8 and its Blaine fineness $458 \mathrm{~m}^{2} / \mathrm{kg}$; according to the XRD analysis, its crystalline content (melilite) was very low. The natural pozzolan $(P)$ had a Blaine fineness of $760 \mathrm{~m}^{2} / \mathrm{kg}$, and an acid volcanic glass content of 93\%; the main crystalline components determined by XRD were quartz and feldspars (anorthite, albite, chabazite and analcite). The chemical composition of the additions is likewise shown in Table 1.

The pozzolan was ground to greater fineness to enhance its initial physical effect and chemical activation, to

Tabla 1 / Table 1

Composición química de los materiales usados

Chemical composition of the materials used

\begin{tabular}{|c|c|c|c|}
\cline { 2 - 4 } \multicolumn{1}{c|}{} & $\begin{array}{c}\text { Cemento Portland } \\
\text { Portland cement }\end{array}$ & $\begin{array}{c}\text { Escoria granulada } \\
\text { Granulated slag }\end{array}$ & $\begin{array}{c}\text { Puzolana natural } \\
\text { Natural pozzolan }\end{array}$ \\
\hline $\mathrm{SiO}_{2} \%$ & 22,44 & 34,27 & 76,87 \\
\hline $\mathrm{Al}_{2} \mathrm{O}_{3} \%$ & 3,40 & 12,68 & 9,80 \\
\hline $\mathrm{Fe}_{2} \mathrm{O}_{3} \%$ & 4,20 & 0,84 & 3,58 \\
\hline $\mathrm{CaO} \%$ & 63,45 & 40,58 & 5,50 \\
\hline $\mathrm{MgO} \%$ & 0,57 & 9,75 & 0,51 \\
\hline $\mathrm{K}_{2} \mathrm{O} \%$ & 1,18 & 0,41 & --- \\
\hline $\mathrm{Na}_{2} \mathrm{O} \%$ & 0,04 & 0,05 & 1,41 \\
\hline $\mathrm{SO}_{3}, \%$ & 2,91 & 0,41 & 2,40 \\
\hline Pérdida por calcinación, \% & 1,82 & 0,11 & \\
\hline Loss on ignition, \% & & & \\
\hline
\end{tabular}


compensar el efecto de dilución inicial del cemento compuesto, la puzolana fue molida a una mayor finura, mientras que la escoria se empleó con la finura comercial. La finura de la puzolana fue establecida de manera que la misma no modificara el consumo de agua del mortero y la elección de moler en mayor medida la puzolana que la escoria se debió a que la primera presenta un mayor grado de molturabilidad $y$, en consecuencia, el proceso de molienda es más económico.

Cementos compuestos estudiados: los cementos con adiciones se obtuvieron por reemplazos variables de cemento CEM I por puzolana natural y escoria granulada de alto horno. Para seleccionar los reemplazos a estudiar y con el fin de evaluar al cemento con adiciones como un sistema de variables interrelacionadas, se adoptó un diseño de experimentos central compuesto centrado (14), en el cual las dos variables experimentales son los porcentajes de reemplazo del cemento por puzolana natural $\left(x_{1}\right)$ y por escoria granulada $\left(x_{2}\right)$. Este sistema permitió definir cuatro cementos binarios y ocho ternarios (Figura 1) para la evaluación de la resistencia mecánica a partir del análisis de las superficies de respuesta. El modelo está representado por la Ecuación [1]. offset the impact of the substitution of the Portland cement in the blend. The standard fineness of the commercial slag used was not altered. Pozzolan fineness was defined in such a way as to ensure that water uptake in the mortar would not be modified. The decision to grind the pozzolan rather than the slag was due to financial considerations, given the readier and therefore less expensive grindability and of the former.

Composite cements studied: the addition-bearing cements were prepared by replacing variable proportions of CEM I with natural pozzolan and granulated blast furnace slag. A central composite experimental design (14) was adopted to select the proportions to be replaced and evaluate the cement with additions as a system with interrelated variables. In this design, the two experimental variables were the percentages of natural pozzolan $\left(x_{1}\right)$ and granulated slag $\left(x_{2}\right)$ replacing the cement. Adopting this approach, four binary and eight ternary cements were manufactured (Figure 1) and their mechanical strength evaluated with response surface analysis. The model is represented by Equation [1].

$$
Y=\beta_{0}+\beta_{1} x_{1}+\beta_{2} x_{2}+\beta_{3} x_{1}+\beta_{4} x_{2}+\beta_{5} x_{1} x_{2}
$$

donde $Y$ es la resistencia a flexión o a compresión a una edad determinada, $x_{1}$ y $x_{2}$ : son las variables experimentales y $\beta_{0} \ldots, \beta_{5}$ : son los coeficientes estimados a partir del método de mínimos cuadrados que se informan en la Tabla 2. El coeficiente $R^{2}$ fue mayor a 0,93 indicando una buena correlación entre los valores experimentales y calculados. La máxima diferencia entre la resistencia a compresión where $Y$ is flexural or compressive strength at a given age, $x_{1}$ and $x_{2}$ are the experimental variables and $\beta_{0} \ldots$, $\beta_{5}$ : are the minimum squares estimated coefficients given in Table 2. The values of coefficient $R^{2}$, over 0.93 in all cases, were indicative of the good correlation between the experimental and calculated results. The maximum difference between experimental and calculated

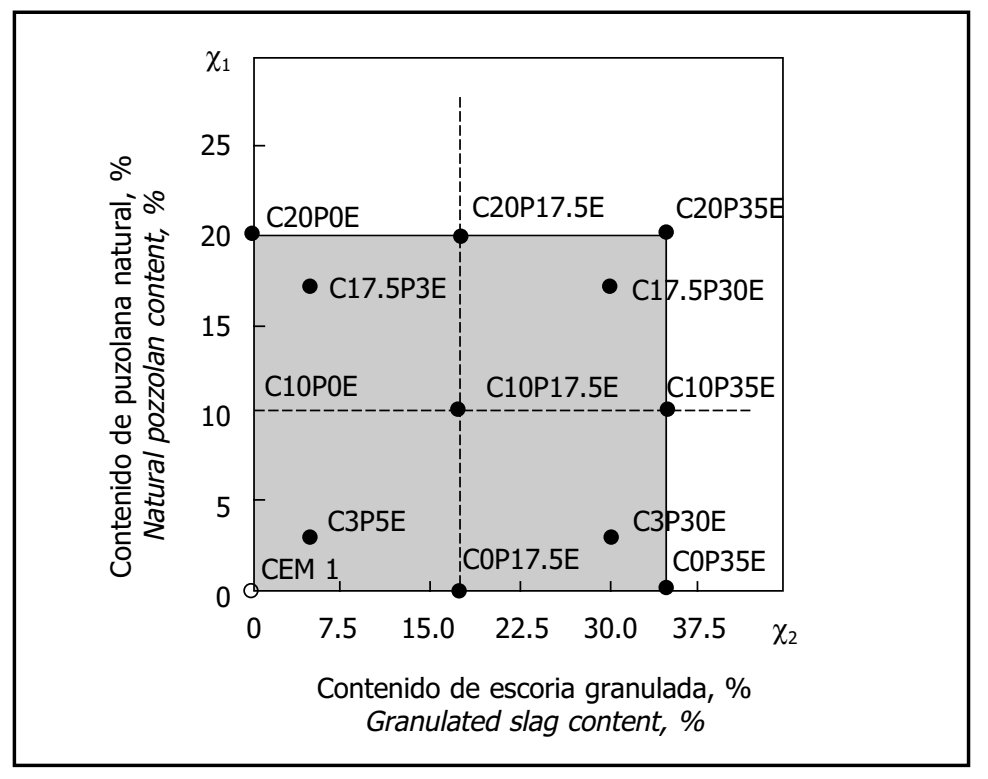

Figura 1. Dominio de los cementos con adiciones estudiados.

Figure 1. Domain of the cements with additions studied. 
experimental y la calculada fue de $\pm 5,3 \%$, en tanto que para la resistencia a flexión fue de $\pm 5,8 \%$.

Proporciones de las mezclas: los morteros se prepararon manteniendo una relación en peso agregado (arena silícea)/material cementante de 3. La relación agua/material cementante $(\mathrm{a} / \mathrm{mc})$ fue de 0,50 para todo el dominio estudiado y la fluidez de los morteros $110 \pm 5 \%$.

Resistencia mecánica: la resistencia a flexión y a compresión de los morteros se evaluó sobre probetas de 40×40x160 mm de acuerdo a la norma EN 196-1 (15). Las edades de ensayos fueron: $2,7,28,90$ y 180 días y los resultados informados en la Tabla 3 corresponden al promedio de tres y seis determinaciones por edad, respectivamente.

Puzolanicidad: la actividad puzolánica por vía química a los 2, 7 y 28 días, se evaluó mediante la comparación de la cantidad de $\mathrm{CH}$ que contenía la disolución acuosa de cada pasta hidratada, con la curva de solubilidad de $\mathrm{CH}$ en una disolución alcalina. La temperatura de ensayo fue de $40{ }^{\circ} \mathrm{C}$. El resultado del ensayo determina actividad puzolánica cuando la concentración de $\mathrm{CH}$ en la disolución acuosa de cada pasta se ubica por debajo de la curva de solubilidad [EN 196-5 (16)].

\section{RESULTADOS}

Consumo de agua: la incorporación de puzolana y escoria no modifica la demanda de agua $(\mathrm{a} / \mathrm{cm}: 0,50)$ del mortero para mantener una fluidez de $110 \pm 5 \%$. Sin embargo, algunas veces se ha argumentado que el empleo conjunto de estas adiciones en la formulación de cementos compuestos puede no ser adecuado, pues esta combinación tiende a incrementar la demanda de agua del cemento para obtener una determinada trabajabilidad (17). Pero en estudios previos (11) se ha determinado que esta puzolana empleada con una finura de Blaine de $760 \mathrm{~m} / \mathrm{kg}$ no modifica el consumo de agua del mortero para obtener una fluidez de $110 \pm 5 \%$. compressive strength was $\pm 5.3 \%$, whereas for flexural strength the largest difference was $\pm 5.8 \%$.

Blend proportions: mortars were prepared with a an aggregate (siliceous sand)/cementitious material ratio of 3 (by weight). The water/cementitious material $(w / c m)$ ratio was 0.50 for the entire domain studied and mortar fluidity was $110 \pm 5 \%$.

Mechanical strength: mortar flexural and compressive strengths were evaluated on $40 \times 40 \times 160 \mathrm{~mm}$ specimens as specified in European standard EN 196-1 (15). Specimens were tested at the following ages: 2, 7, 28, 90 and 180 days; the results shown in Table 3 are the mean of three and six replicates per age, respectively.

Pozzolanicity: chemical pozzolanicity was evaluated at 2, 7 and 28 days by comparing the $\mathrm{CH}$ concentration in the aqueous solution of each hydrated paste to the solubility curve for $\mathrm{CH}$ in an alkaline solution. The test temperature was $40^{\circ} \mathrm{C}$. Specimens test positive for pozzolanic activity when $\mathrm{CH}$ concentration in the aqueous solution is beneath the solubility curve [EN 196-5 (16)].

\section{RESULTS}

Water uptake: water uptake (w/cm: 0.50) did not have to be altered to maintain fluidity at $110 \pm 5 \%$ when pozzolan and slag were added. Nonetheless, it has been sustained on occasion that the joint use of such additions may not be suitable in composite cements, for this combination tends to raise the water needed to reach a certain workability (17). But previous research (11) showed that use of this pozzolan with a Blain fineness of 760 $\mathrm{m} / \mathrm{kg}$ does not modify the amount of water required to attain mortar fluidity of $110 \pm 5 \%$.

\section{Tabla 2 / Table 2}

Coeficientes estimados a partir del método de mínimos cuadrados y $\mathrm{R}^{2}$ Minimum squares-estimated coefficients and $R^{2}$ values

\begin{tabular}{|c|c|c|c|c|c|c|c|c|c|c|c|c|c|c|}
\hline \multirow[t]{2}{*}{$\begin{array}{l}\text { Edad, días } \\
\text { Age, days }\end{array}$} & \multicolumn{6}{|c|}{$\begin{array}{l}\text { Coeficientes resistencia a compresión } \\
\text { Compressive strength coefficients }\end{array}$} & \multicolumn{8}{|c|}{$\begin{array}{l}\text { Coeficientes resistencia a flexión } \\
\text { Flexural strength coefficients }\end{array}$} \\
\hline & $\beta_{0}$ & $\beta_{1}$ & $\beta_{2}$ & $\beta_{3}$ & $\beta_{4}$ & $\beta_{5}$ & $\mathbf{R}^{\mathbf{2}}$ & $\beta_{0}$ & $\beta_{1}$ & $\beta_{2}$ & $\beta_{3}$ & $\beta_{4}$ & $\beta_{5}$ & $\mathbf{R}^{\mathbf{2}}$ \\
\hline 2 & 16.8 & 29.1 & -8.8 & -179.9 & -21.1 & -23.8 & 0.96 & 3.4 & 4.0 & -1.4 & -27.9 & -6.0 & -2.2 & 0.95 \\
\hline 7 & 32.1 & 26.6 & -13.5 & -189.2 & -5.3 & -11.4 & 0.95 & 5.7 & -3.5 & -1.3 & 11.0 & -3.1 & -4.9 & 0.93 \\
\hline 28 & 43.2 & 41.3 & 7.2 & -204.0 & -24.9 & -28.7 & 0.97 & 6.6 & 8.3 & 1.1 & -24.1 & 0,3 & -16.3 & 0.93 \\
\hline 90 & 46.9 & 71.0 & 2.2 & -205.5 & 59.7 & -126.7 & 0.94 & 7.7 & 12.6 & 2.2 & -42.8 & -2.4 & -8.2 & 0.97 \\
\hline 180 & 48.3 & 83.1 & 16.5 & -162.2 & 30.6 & -113.0 & 0.99 & 8.2 & 10.2 & 4.5 & -25.1 & -6.4 & -10.7 & 0.95 \\
\hline
\end{tabular}


Tabla 3 / Table 3

Valores experimentales de la resistencia a compresión y a flexión de los morteros Experimental values for mortar compressive and flexural strength

\begin{tabular}{|c|c|c|c|c|c|c|c|c|c|c|}
\hline \multirow[t]{2}{*}{$\begin{array}{l}\text { Mortero } \\
\text { Mortar type }\end{array}$} & \multicolumn{6}{|c|}{$\begin{array}{l}\text { Resistencia a compresión, } \mathrm{MPa} \\
\text { Mean compressive strength, } \mathrm{MPa}\end{array}$} & \multicolumn{4}{|c|}{$\begin{array}{c}\text { Resistencia a flexión, } \mathrm{MPa} \\
\text { Flexural strength, } \mathrm{MPa}\end{array}$} \\
\hline & $2 \mathrm{~d}$ & $7 \mathrm{~d}$ & $28 \mathrm{~d}$ & $90 \mathrm{~d}$ & $180 \mathrm{~d}$ & $2 \mathrm{~d}$ & $7 \mathrm{~d}$ & $28 \mathrm{~d}$ & $90 \mathrm{~d}$ & $180 \mathrm{~d}$ \\
\hline CEM I & 16.6 & 31.7 & 43.0 & 46.4 & 486 & 3.3 & 5.6 & 6.6 & 7.6 & 8.1 \\
\hline C10P0E & 18.7 & 33.5 & 45.4 & 52.2 & 55.1 & 3.4 & 5.5 & 7.2 & 8.5 & 9.0 \\
\hline C2OP0E & 14.8 & 29.5 & 43.1 & 53.4 & 58.4 & 3.2 & 5.4 & 7.4 & 8.6 & 9.3 \\
\hline COP17.5E & 14.4 & 28.8 & 43.9 & 49.8 & 52.0 & 2.8 & 5.2 & 6.9 & 8.1 & 8.9 \\
\hline COP35E & 11.2 & 26.7 & 42.8 & 54.9 & 58.2 & 2.1 & 4.9 & 7.0 & 8.2 & 9.0 \\
\hline C3P5E & 17.1 & 32.5 & 44.5 & 49.4 & 51.2 & 3.5 & 5.7 & 6.9 & 8.2 & 8.8 \\
\hline C17.5P3E & 15.9 & 30.1 & 44.7 & 51.3 & 57.5 & 2.9 & 5.2 & 7.3 & 8.6 & 9.3 \\
\hline C10P17.5E & 15.0 & 29.8 & 45.3 & 51.2 & 57.0 & 3.1 & 5.2 & 7.2 & 8.8 & 9.4 \\
\hline C20P17.5E & 13.4 & 27.3 & 42.7 & 50.7 & 58.7 & 2.6 & 5.0 & 6.9 & 8.5 & 9.4 \\
\hline C3P30E & 13.2 & 29.4 & 43.6 & 53.0 & 56.8 & 2.6 & 5.1 & 7.1 & 8.3 & 9.2 \\
\hline C10P35E & 11.5 & 26.0 & 43.7 & 56.1 & 60.8 & 2.2 & 4.3 & 7.0 & 8.8 & 9.3 \\
\hline C17.5P30E & 9.5 & 25.9 & 42.5 & 53.6 & 60.1 & 2.0 & 4.3 & 6.8 & 8.7 & 9.3 \\
\hline C20P35E & 8.6 & 24.0 & 41.0 & 51.5 & 59.6 & 1.8 & 4.4 & 6.7 & 8.5 & 9.4 \\
\hline
\end{tabular}

Adicionalmente, la utilización de una puzolana de la misma procedencia y características similares (93\% de vidrio volcánico ácido, finura Blaine: $630 \mathrm{~m}^{2} / \mathrm{kg}$ ) en hormigones con alto contenido de puzolana ( 0 a $60 \%$ ) produjo una mejora en la consistencia, la cohesión y la terminación del hormigón a medida que se incrementaba el porcentaje de adición incorporado (18). Esto indica que si las adiciones no presentan una forma muy desfavorable, ni una alta absorción y no reaccionan durante las primeras horas de hidratación, el aumento en la cantidad de agua dado por el incremento de su finura puede ser compensado por la menor cantidad de agua químicamente combinada.

Resistencia mecánica: las Figuras 2 y 3 muestran las curvas de isorrespuestas de la resistencia a compresión y a flexión de los morteros, en éstas se puede observar con facilidad la influencia que ejercen los distintos cementos binarios sobre estas propiedades.

En este sentido, para cementos binarios con hasta $12 \%$ de puzolana natural $\left(x_{1}\right)$ (Figuras $2 \mathrm{a}$ y $\mathrm{b}$ ) se registra un incremento en la resistencia a compresión con respecto a la obtenida por el mortero CEM I. Para valores de $x_{1}$ entre 0 y $20 \%$ a 28 días (Figura 2 c) se alcanza un nivel resistente igual o mayor al del mortero CEM I. A edades más avanzadas (Figuras $2 \mathrm{~d}$ y e), se produce un cambio en las curvas de isorrespuestas indicando que a medida que aumenta el valor de $x_{1}$ se produce un incremento de la resistencia del cemento binario con puzolana natural. Así, a 180 días cuando $x_{1}$ alcanza al $20 \%(58,4 \mathrm{MPa})$, se produce un aumento de resistencia del orden del $20 \%$ con respecto a la registrada por el mortero CEM I (48,6 $\mathrm{MPa})$.
Moreover, when a pozzolan from the same source and with similar characteristics (93\% acid volcanic glass, Blain fineness of $630 \mathrm{~m}^{2} / \mathrm{kg}$, ) was used in concretes with a high pozzolan content ( 0 to 60\%), better consistency, bonding and concrete finish were obtained at higher replacement rates (i.e., higher percentages of the addition) (18). This suggests that if the additions do not have a very unfavourable shape or high absorption rates and do not react during the first few hours of hydration, the increase in the water uptake prompted by greater fineness may be offset by the smaller amount of chemically combined water.

Mechanical strength: the iso-response curves for mortar compressive and flexural strength in Figures 2 and 3 clearly illustrate the effect of the different binary cements on these properties.

In this regard, compressive strength is observed to be higher in binary cements with up to $12 \%$ of natural pozzolan $\left(x_{1}\right)$ (Figures 2a and $b$ ) than in CEM I mortar. At 28 days, for values of $x_{1}$ of from 0 to $20 \%$ (Figure $2 c$ ), strength levels are at least equal to the levels recorded for CEM I. The change in the iso-response curves at older ages (Figures $2 d$ and e) is an indication that as the value of $x_{1}$ grows, the strength of natural pozzolan-containing binary cement rises. At 180 days and an $x_{1}$ of $20 \%(58.4 \mathrm{MPa})$, for instance, strength is on the order of $20 \%$ higher than in CEM I mortar (48.6 MPa). 


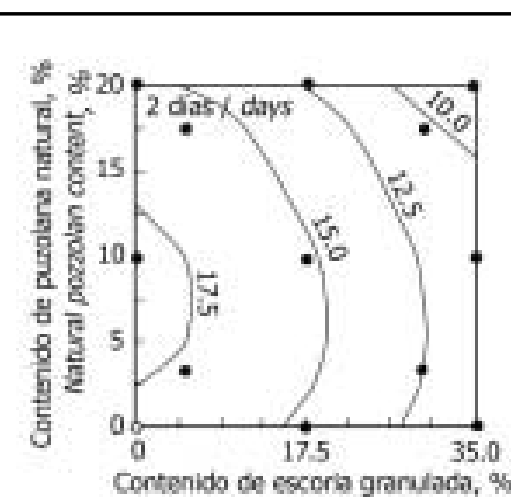

a)

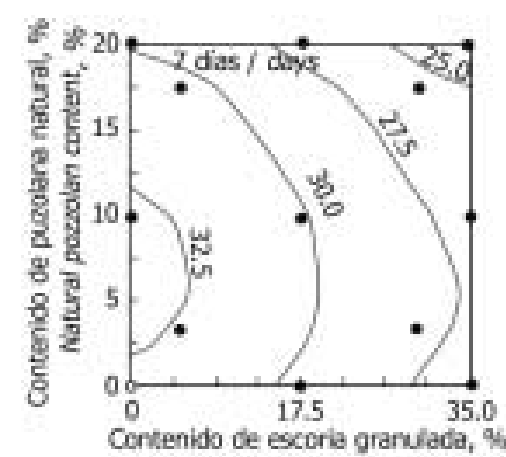

b) Granulated slag canterx, \%

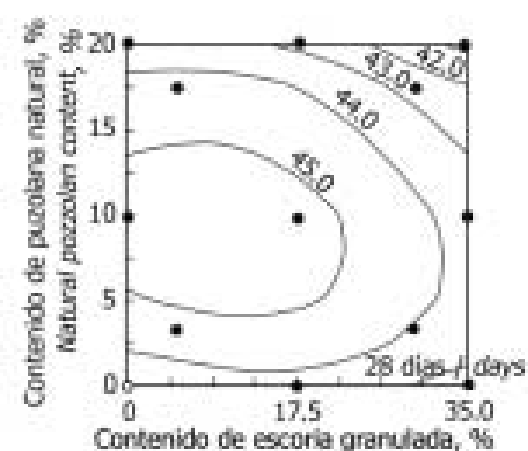

c) Granclated slag cantent, \%

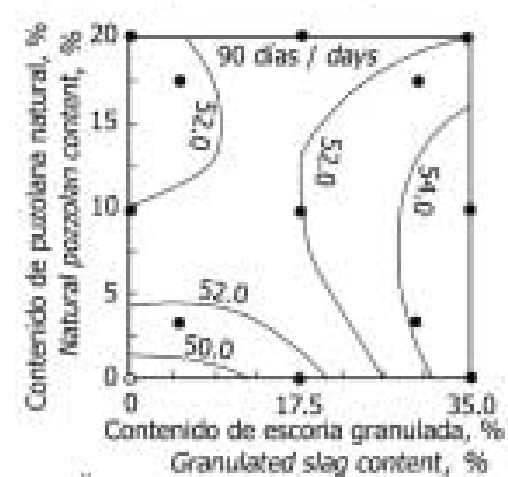

d)

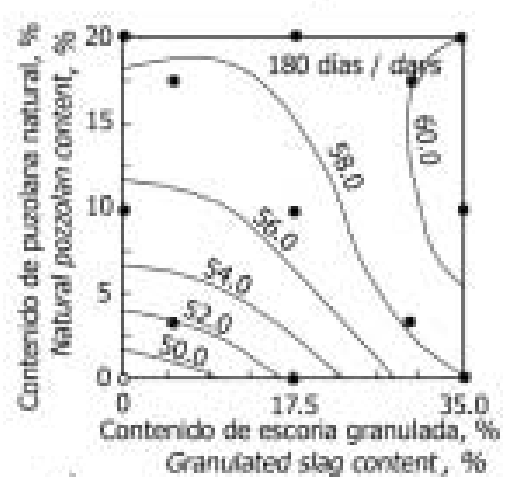

e)

Figura 2. Curvas de isorrespuestas de la resistencia a compresión de los morteros: a) 2 días, b) 7 días, c) 28 días, d) 90 días y e) 180 días. Figure 2. Mortar compressive strength iso-response curves: a) 2 days, b) 7 days, c) 28 days, d) 90 days and e) 180 days.

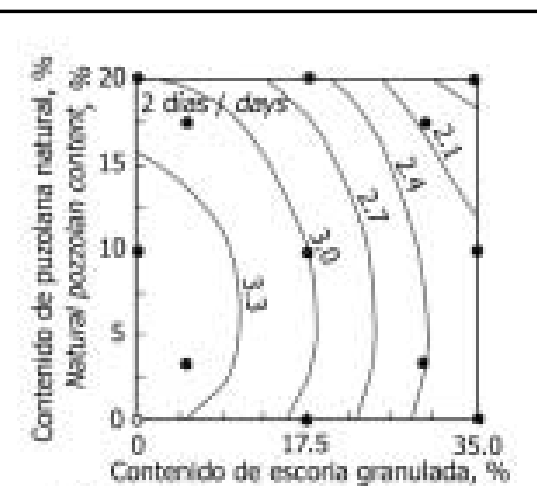

a)

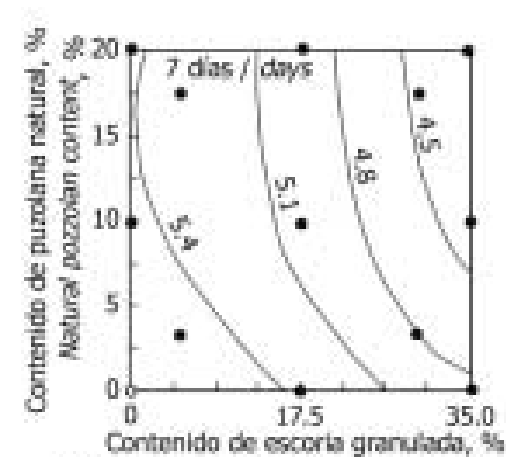

b)

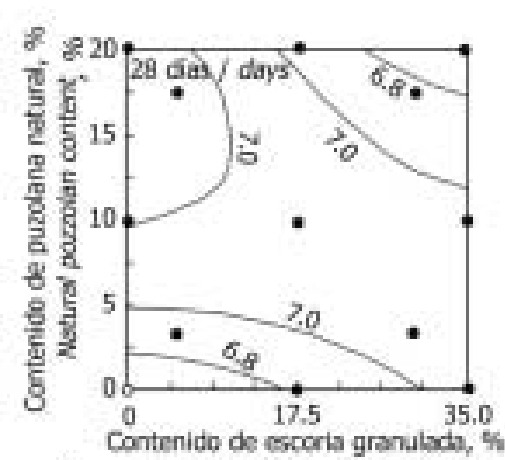

c)

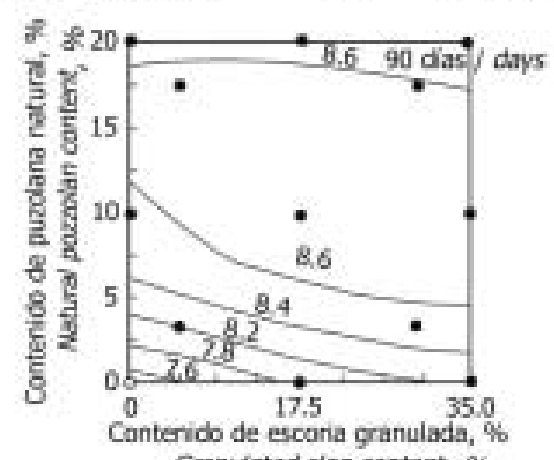

d)

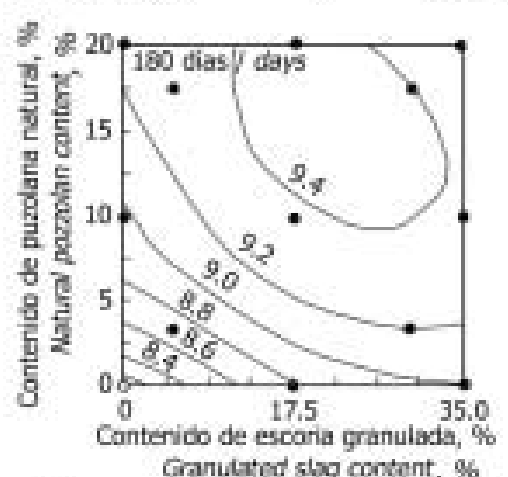

e)

Figura 3. Curvas de isorrespuestas de la resistencia a flexión de los morteros: a) 2 días, b) 7 días, c) 28 días, d) 90 días y e) 180 días. Figure 3. Mortar flexural strength iso-response curves: a) 2 days, b) 7 days, c) 28 days, d) 90 days and e) 180 days. 
Cuando se analiza el contenido de escoria granulada $\left(\chi_{2}\right)$ en el cemento binario, puede observarse que en las primeras edades ( 2 y 7 días) con hasta $16 \%$ de escoria se registra un nivel resistente similar al obtenido por el mortero CEM I (Figuras $2 a$ y b). A 28 días, con valores de $\chi_{2}$ comprendidos entre 0 y $30 \%$, se alcanza la resistencia registrada por el mortero CEM I (Figura $2 c$ ). Sin embargo, a edades más avanzadas (Figuras $2 \mathrm{~d}$ y e), a medida que se incrementa el valor de $\chi_{2}$, se registran mayores resistencias, por ejemplo a 180 días con $\chi_{2}=35 \%(58,2$ $\mathrm{MPa}$ ) se alcanza una resistencia $20 \%$ mayor que la obtenida por el mortero CEM I (48,6 MPa).

Para los cementos ternarios, las máximas resistencias se pueden obtener para valores de $\chi_{1}$ y $\chi_{2}$ de hasta 12 y $4 \%$, a 2 y 7 días (Figuras $2 a$ y b). Pero a estas edades el nivel resistente del mortero CEM I puede alcanzarse con valores de $\chi_{1}$ y $\chi_{2}$ considerablemente mayores. A 28 días el punto estacionario (punto de máxima resistencia) queda determinado por $\chi_{1}=10 \%$ y $\chi_{2}=9 \%(45,5 \mathrm{MPa})$, aun así la curva de isorrespuesta de $45 \mathrm{MPa}$ define una amplia gama de combinaciones de puzolana y de escoria capaces de registrar una resistencia mayor a la obtenida por el mortero CEM I.

Además, a esta edad una resistencia mayor o igual a $42,5 \mathrm{MPa}$ puede alcanzarse con valores de $\chi_{1}$ y $\chi_{2}$ de 20 y $26 \%$, y 17,5 y $35 \%$, respectivamente. A 90 días, las curvas de isorrespuestas presentan la forma de una silla de montar (paraboloide hiperbólico), indicando que, a edades más avanzadas, las superficies de respuestas presentarán un cambio en la pendiente con respecto a las registradas a edades tempranas. Así, a 180 días, las curvas de isorrespuestas de máximas resistencias giran y se trasladan a valores de $\chi_{1}$ y $\chi_{2}$ mayores y a esta edad una resistencia por lo menos $24 \%$ mayor (> $60 \mathrm{MPa}$ ) a la registrada por el mortero CEM I pueden alcanzarse para valores de $\chi_{1}$ entre 6 y $20 \%$ y de $\chi_{2}$ de $35 \%$.

Una evolución de la resistencia, similar o mayor a la registrada por el mortero CEM I, puede alcanzarse con valores de $\chi_{1}+\chi_{2}$ en el orden del $30 \%$ a 2 y 7 días, del $40 \%$ a 28 días y del $55 \%$ a 90 y 180 días. Esto se traduce en una disminución de clínker Portland para elaborar una tonelada de cemento compuesto con similar evolución de la resistencia del mortero.

La evolución de la resistencia a flexión presenta una tendencia similar a la registrada por la resistencia a compresión. A 2 días (Figura 3a), una resistencia a flexión similar a la obtenida con el mortero CEM I se registra para valores de $\chi_{1}$ en el orden de $15 \%$ y bajos valores de $\chi_{2}(<8 \%)$; mientras que a 7 días esta resistencia puede lograrse con $\chi_{1}=20 \%$ y $\chi_{2}=17 \%$ (Figura $3 \mathrm{~b}$ ). A estas edades, al igual de lo que sucede con la resistencia a compresión, para un determinado valor de $\chi_{1}$ a
An analysis of granulated slag content $\left(\chi_{2}\right)$ in binary cement reveals that at the younger ages ( 2 and 7 days) strength values for blends with up to $16 \%$ slag are similar to the values found for CEM I mortar (Figures 2a and b). After 28 days, the strength reached in blends with $\chi_{2}$ values of from 0 to $30 \%$ is the same as recoded for CEM I mortar (Figure 2c). And at older ages (Figures $2 d$ and e), strength rises with the value of $\chi_{2}$ : at 180 days, for instance, the strength recorded in blends with $\chi_{2}=35 \%$ (58.2 MPa) is $20 \%$ higher than obtained for CEM I mor$\operatorname{tar}(48.6 \mathrm{MPa})$.

In ternary cements, the highest 2- and 7-day strength is reached with $\chi_{1}$ and $\chi_{2}$ values of up to 12 and $4 \%$ (Figures $2 a$ and $b$ ). At these ages, moreover, mortar strength equivalent to CEM I can be reached with substantially larger values of $\chi_{1}$ and $\chi_{2}$. The 28-day stationary point (peak strength) is $\chi_{1}=10 \%$ and $\chi_{2}=9 \%$ (45.5 MPa), although the $45 \mathrm{MPa}$ iso-response curve defines a wide range of combinations of slag and pozzolan able to reach strengths greater than recorded for CEM I mortar.

Indeed, at this age strengths greater than or equal to $42.5 \mathrm{MPa}$ can be attained with $\chi_{1}$ and $\chi_{2}$ values of 20 and $26 \%$ and 17.5 and $35 \%$, respectively. The saddle (hyerbolic paraboloid) shape adopted by the 90-day isoresponse curves is an indication that at older ages the slope of the response surfaces differs from the slope found at earlier ages. And at 180 days, the maximum strength iso-response curves veer to higher $\chi_{1}$ and $\chi_{2}$ values; at that age strength at least $24 \%$ greater (>60 MPa) than recorded for CEM I mortar is attained with $\chi_{1}$ values of from 6 to $20 \%$ and $\chi_{2}$ values of $35 \%$.

Strength development similar to or greater than observed in CEM I mortar can be attained with values of $\chi_{1}+$ $\chi_{2}$ on the order of $30 \%$ at 2 and 7 days, $40 \%$ at 28 days and $55 \%$ at 90 and 180 days. This translates into a reduction in the amount of Portland clinker needed to manufacture a tonne of composite cement comparable in strength to pure Portland cement.

The pattern for flexural strength development is similar to the pattern for compressive strength. After 2 days (Figure 3a), flexural strength similar to CEM I mortar values is attained for $\chi_{1}$ values on the order of $15 \%$ and small values of $\chi_{2}(<8 \%)$; at 7 days, however, such strength can be attained with $\chi_{1}=20 \%$ and $\chi_{2}=17 \%$ (Figure $3 b$ ). At these ages, as in compressive strength, for a given value of $\chi_{1}$, flexural strength declines as the value of $\chi_{2}$ climbs, indicating that in ternary cement this 
medida que el valor de $\chi_{2}$ aumenta la resistencia a flexión diminuye, indicando que este parámetro en el cemento ternario depende principalmente del contenido de escoria incorporado. A 28 días, resistencias mayores al mortero CEM I $(6,6 \mathrm{MPa})$ pueden obtenerse para valores de $\chi_{1}$ entre 3 y $20 \%$ y de $\chi_{2}$ entre 22,5 y $35 \%$ (Figura $3 c)$, mientras que la máxima resistencia $(8,8 \mathrm{MPa}$, punto estacionario) se encuentran para valores de $\chi_{1}+\chi_{2}: 12+27 \%$, resultando un $16 \%$ mayor a la registrada por el mortero CEM I (7,6 MPa). A 90 días, la resistencia a flexión del cemento ternario depende principalmente del contenido de escoria incorporado, debido a que el contorno de las curvas de isorrespuestas tienden a ser paralelas al valor de $\chi_{2}$. A 180 días, cualquier valor de $\chi_{1}$ y $\chi_{2}$ produce una resistencia superior a la registrada por el mortero CEM I y el punto estacionario se encuentra en valores de $\left(\chi_{1}, \chi_{2}\right)(16,23)$, alcanzando una resistencia $17 \%(9,48 \mathrm{MPa})$ mayor a la obtenida por el mortero CEM I (8,1 MPa).

\section{DISCUSIÓN}

En trabajos previos $(19,20)$ se ha demostrado que, durante las primeras edades de la hidratación, la incorporación aislada de puzolana natural molida aumenta la cantidad de agua no evaporable del cemento Portland. Estos resultados también son corroborados por el exceso de alcalinidad evaluado por vía química a la edad de 2 días como se muestra en la Figura 4a; en la que puede observarse que los puntos representativos de las muestras C10P0E y C20P0E se encuentran ubicados en la zona de sobresaturación. La mayor cantidad de $\mathrm{CH}$ que se ha registrado en estos cementos indica que la puzolana produce una aceleración en la reacción de los silicatos del clínker Portland. El incremento de la parameter depends primarily on slag content. Strengths greater than found in CEM I mortar (6.6 MPa) can be reached at 28 days for $\chi_{1}$ values of from 3 to $20 \%$ and $\chi_{2}$ values of from 22.5 to $35 \%$ (Figure $3 c$ ), whereas the peak strength (8.8 MPa, stationary point) is found for $\chi_{1}+\chi_{2}$ values of $12+27 \%$. The strength observed is $16 \%$ greater than recorded for CEM I mortar (7.6 MPa). At 90 days the flexural strength of ternary cement depends primarily on the slag content, inasmuch as the shape of the iso-response curves tend to be parallel to the $\chi_{2}$ values. After 180 days, any value of $\chi_{1}$ and $\chi_{2}$ yields higher strength than CEM I mortar and the stationary point is found at $\left(\chi_{1}, \chi_{2}\right)$ values of $(16,23)$, where strength $(9.48 \mathrm{MPa})$ is $17 \%$ higher than obtained for CEM I mortar (8.1 MPa).

\section{DISCUSSION}

Prior research $(19,20)$ showed that at early hydration ages, the separate inclusion of ground natural pozzolan increases the amount of non-evaporable water in Portland cement. These results are corroborated by the excess alkalinity found by chemical methods in 2-day mortars, as shown in Figure 4a. Here the points representing samples C1OPOE and C2OPOE are located in the supersaturated zone. The higher amount of $\mathrm{CH}$ found in these cements is an indication that the pozzolan expedites the reaction time of Portland clinker silicates. Initial hydration takes place faster because the inclusion of finely ground materials generates a larger number of nuclei where $\mathrm{CH}$ crystals can begin to grow (21). This

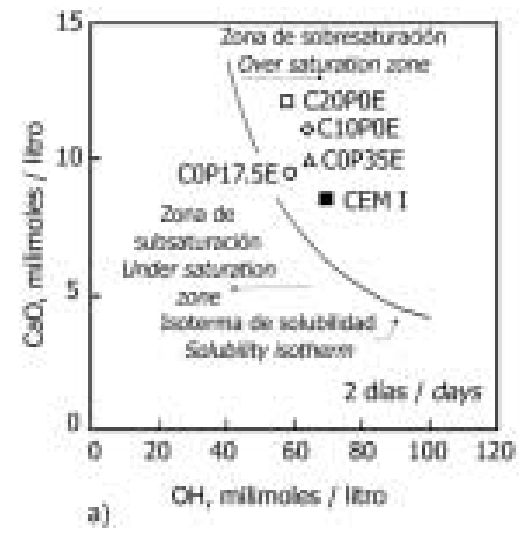

a)

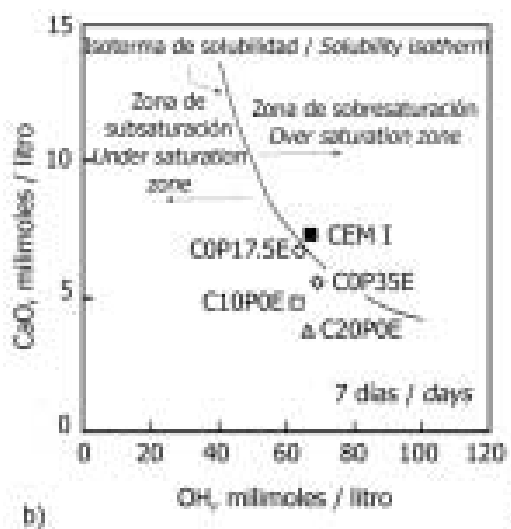

b)

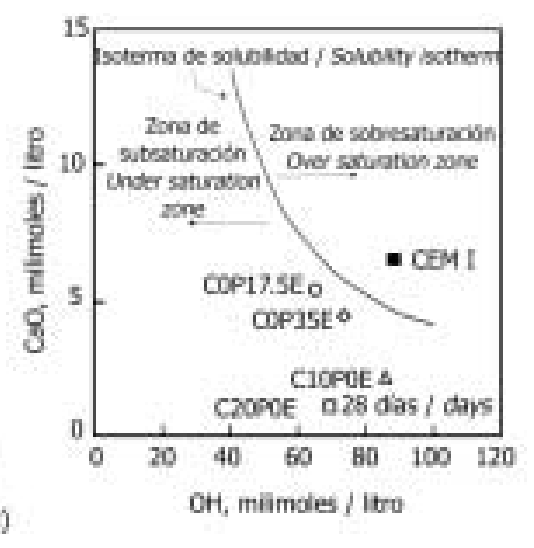

Figura 4. Actividad puzolánica por vía química de los cementos: a) 2 días, b) 7 días y c) 28 días.

Figure 4. Chemical pozzolanic activity in cement: a) 2 days, b) 7 days and c) 28 days. 
hidratación inicial se produce debido a que la incorporación de materiales finamente molidos genera una mayor cantidad de núcleos a partir de los cuales comienza el crecimiento de los cristales de $\mathrm{CH}$ (21). Este comportamiento, denominado efecto filler produce un cambio en la microestructura y un aumento la resistencia mecánica.

Adicionalmente, a edades tempranas ( 2 y 7 días) para todo el dominio de puzolana estudiado, se produce una resistencia similar o algo superior a la obtenida por el CEM I. Es decir, que el aumento en el efecto filler sumado al incremento en la velocidad de la reacción de la puzolana dado por la activación física (Figura 4b, los puntos representativos se ubican en la zona de subsaturación indicando que el CH está siendo consumido por la reacción puzolánica) alcanzan para contrarrestar el efecto de dilución del cemento Portland. Con el avance de la hidratación y la mayor reacción de la puzolana (Figura 4c) se produce una microestructura más densa por el consumo de $\mathrm{CH}$ y la generación de CSH [refinamiento de granos y poros (6)], y entonces, a medida que se incrementa el contenido de puzolana, aumenta la resistencia del mortero.

En lo concerniente al cemento binario con escoria granulada, también se ha demostrado previamente $(9,10)$ que el efecto filler produce un aumento del agua no evaporable hasta los 3 días y este comportamiento también puede observarse en la Figura $4 a$.

Sin embargo, debido a la menor velocidad de hidratación de la escoria (4) con respecto a la del cemento Portland y a la de la puzolana (dada por su mayor finura), los cementos con esta adición presentan una disminución inicial de la resistencia para altos porcentajes de reemplazos, pues, a pesar de que la escoria ha comenzado a reaccionar a los 7 días (Figura 4b), el efecto de dilución resulta más importante para contenidos de reemplazos elevados. Con la posterior reacción de la escoria este comportamiento tiende a revertirse y la resistencia aumenta conforme se incrementa su contenido en el cemento Portland.

Adicionalmente, en la Figura 4 puede verse que la puzolana molida produce un mayor efecto filler que la escoria y que la cantidad de $\mathrm{CH}$ consumida por la puzolana es mayor que la que consume la escoria. Esto pone en evidencia, por una parte, la activación física de la puzolana, y por otra, que la escoria, durante su reacción, no consume demasiada cantidad del $\mathrm{CH}$ producido por la hidratación de los silicatos de calcio del cemento Portland.

En función de lo expuesto, la resistencia inicial del mortero con cemento compuesto será función principalmente del contenido de puzolana natural (Figuras 2a y 3a), mientras que la resistencia final será función de la cantidad development, known as the filler effect, changes the microstructure and raises mechanical strength.

Moreover, at early ages ( 2 and 7 days), strength is similar or slightly higher than observed for CEM I across the entire domain of the pozzolan studied. In other words, the enhanced filler effect, coupled with the increase in the pozzolan reaction rate as a result of physical activation (Figure $4 b$, the points in question are located in the supersaturation zone, indicating that $\mathrm{CH}$ is being taken up in the pozzolanic reaction), suffice to offset the effect of reducing the proportion of Portland cement. As hydration and the pozzolan reaction advance (Figure $4 c$ ), the microstructure grows denser due to the uptake of $\mathrm{CH}$ and generation of $\mathrm{CSH}$ [grain and pore size refinement (6)]. Consequently, as the pozzolan content increases, mortar strength rises.

Previous studies $(9,10)$ with granulated slag binary cement also showed that the filler effect increases the amount of non-evaporable water up to the third day, an effect also observed in Figure $4 a$.

Inasmuch as slag has a slower hydration rate (4) than Portland cement and (finely ground) pozzolan, cements with this addition exhibit lower initial strength at high replacement rates. Indeed, even though the slag begins to react after 7 days (Figure 4b), at high replacement rates the effect of substituting Portland cement continues to prevail. This behaviour tends to revert as the slag continues to react and strength grows as its content in the Portland cement rises.

It may likewise be deduced from Figure 4 that ground pozzolan generates a greater filler effect than slag and the amount of $\mathrm{CH}$ taken up by the pozzolan is greater than the uptake in slag. This is an indication, on the one hand, of the physical activation of the pozzolan, and on the other that slag does not take up very large amounts of the $\mathrm{CH}$ generated by the hydration of the calcium silicates in Portland cement.

In light of the foregoing, the initial strength of mortar made with composite cement may be said to depend primarily on the natural pozzolan content (Figures $2 a$ and $3 a)$, whereas the final strength reached depends on the 
incorporada de ambas adiciones (Figuras 2e y $3 e$ ). Es decir, que la pérdida de resistencia inicial comúnmente ocasionada por la incorporación de puzolana o escoria en finuras convencionales (en el orden de los 350 a $450 \mathrm{~m}^{2} / \mathrm{kg}$ ) puede ser subsanada a partir de la activación física de alguna de ellas y en este caso en particular de la puzolana natural.

\section{CONCLUSIONES}

De acuerdo a los resultados obtenidos en los morteros elaborados con cemento Portland, puzolana natural molida $(0-20 \%)$ y escoria granulada de alto horno (0-35\%) se puede concluir que:

- La activación física de la puzolana natural mejora la resistencia a edades tempranas del cemento ternario, mientras que la acción conjunta de ambas adiciones contribuye con la resistencia a edades más avanzadas, esto hace que el cemento ternario presente un adecuado desarrollo resistente.

- La utilización de cementos compuestos conteniendo una adecuada combinación de cemento Portland y dos adiciones produce un empleo más racional de los recursos naturales sin comprometer la resistencia a compresión o flexión del mortero.

\section{AGRADECIMIENTOS}

Los autores del trabajo quieren agradecer a las empresas Cementos Avellaneda S.A., Loma Negra CIASA y Grupo Minetti por la provisión de los materiales para realizar el estudio. total amount of the two additions replacing the cement (Figures $2 e$ and $3 e$ ). In other words, the decline in initial strength commonly caused by the addition of pozzolan or slag ground to conventional fineness (on the order of 350 to $450 \mathrm{~m}^{2} / \mathrm{kg}$ ) can be offset by the physical activation of one of them, in the present case the natural pozzolan.

\section{CONCLUSIONS}

Further to the results obtained for mortars made with Portland cement, (0-20\%) ground natural pozzolan and (0-35\%) granulated blast furnace slag, it may be concluded that:

- Physical activation of the natural pozzolan improves early age strength in ternary cement, whereas the joint action of the two additions contributes to strength at older ages; i.e., ternary cement exhibits appropriate strength development.

- The use of composite cements with a suitable combination of Portland cement and two additions provides for a more rational use of natural resources without compromising the compressive or flexural strength of mortars.

\section{ACKNOWLEDGEMENTS}

The authors wish to thank Cementos Avellaneda S.A., Loma Negra CIASA and Grupo Minetti for supplying the material used in this study.

\section{BIBLIOGRAFÍA/BIBLIOGRAPHY}

(1) Bonavetti, V. L.: "Hormigón con elevado contenido de adiciones (Green Concrete)", Hormigones Especiales. Editado por la Asociación Argentina de Tecnología del Hormigón (2004), pp. 97-142.

(2) Lea, F. M.: The Chemistry of Cement and Concrete, Chemical Publishing Company, 1971.

(3) Isaia, G. C., Gastaldini, A. L. G. y Moraes, R.: "Physical and pozzolanic action of mineral additions on the mechanical strength of high-performance concrete", Cement and Concrete Composites, no 25 (1) (2003), pp. 69-76.

(4) ACI 233: Ground Granulated Blast-Furnace Slag as a Cementitious Constituent in Concrete. ACI Manual of Concrete Practice. Part 1. Materials and General Propierties of Concrete, 1998.

(5) ACI 232: Use of natural pozzolans in concrete. ACI Manual of Concrete Practice. Part 1. Materials and General Propierties of Concrete, 1998.

(6) Mehta, P. K.: "Pozzolanic and cementitious by-products in concrete-another look", ACI SP no 114, vol. 1 (1989), pp. 1-43.

(7) Escalante-García, J. I., Sharp, J. H.: "The microstructure and mechanical properties of blended cements hydrated at various temperatures", Cement and Concrete Research, no 31 (5) (2001), pp. 695-702.

(8) Fernández-Jiménez, A., Puertas, F. y Arteaga, A.: "Determination of kinetic equations of alkaline activation of blast furnace slag by means of calorimetric data", Journal Thermal Analyses, no 52 (1998), pp. 945-955.

(9) Menéndez, G., Bonavetti, V. L. y Irassar, E. F.: "Stength development of ternary blended cement with limestone filler and blast-furnace slag", Cement and Concrete Composites, no 25 (1) (2003), pp. 61-67. 
(10) Carrasco, M. F., Menéndez, G., Bonavetti, V. L. y Irassar, E. F.: "Strength Optimization of 'Tailor Made Cement' with Limestone Filler and Blast Furnace Slag", Cement and Concrete Research, no 35 (7) (2005), pp. 1324-1331.

(11) Menéndez, G., Bonavetti, V. L., Donza, H., Rahhal, V. y Irassar, E. F.: "Cementos a medida con filler calcáreo y puzolana", Proc. 15a Reunión Técnica de la Asociación Argentina del Hormigón. Santa Fé, Argentina. En CD, 2003, 8 pp.

(12) European Standard for Common Cements: EN 197-1: 2000. Composition, Specifications and Conformity Criteria for Common Cements.

(13) Ellebrock, H. G., Sprung, S. y Kuhlmann, K.: "Particle size Distribution and Properties of Cement. Part III: Influence of theGrinding Process", Zement-Kalk-Gips, no 43 (1) (1990), pp. 13-19.

(14) Montgomery, D. y Runger, G.: Probabilidad y Estadística Aplicadas a la Ingeniería, México, Mc Graw Hill, 1996, p. 787.

(15) European Standard for Common Cements: EN 196-1: 2000. Determination of Strength.

(16) European Standard for Common Cements: EN 196-5: 2000. Pozzolanicity Test for Pozzolanic Cements.

(17) Opoczky, L. y Tamas, F. D.: Multicomponent composite cement. Advances in Cement Technology: Chemistry, Manufacture and Testing. Ed. S. N. Ghosh. New Delhi, India (2002), pp. 559-594.

(18) Giaccio, G., Giovanbattista, A. y Zerbino, R.: "Propiedades de los hormigones elaborados con alto volumen de puzolanas naturales", Hormigón, no 20 (1991), pp. 5-14.

(19) Bonavetti, V. L., Rahhal, V. F. y Irassar, E. F.: "Evolución de la hidratación de cementos con adiciones", Materiales de Construcción, no 52 (268) (2002), pp. 57-64.

(20) Rahhal, V. F.: Caracterización de las adiciones puzolánicas por calorimetría de conducción. Tesis Doctoral. Universidad Politécnica de Madrid, España, 2002, 608 pp.

(21) Zhang, C., Wang, A., Tang, M. y Liu, X.: "The Filling Role of Pozzolanic Material", Cement and Concrete Research, 26 (6) (1996), pp. 943-947. 